\title{
Continued Outbreak of Ceftriaxone-Resistant Salmonella enterica Serotype Typhi across Pakistan and Assessment of Knowledge and Practices among Healthcare Workers
}

\author{
Summiya Nizamuddin, ${ }^{1} \dagger$ Carly Ching, ${ }^{2} \dagger$ Rashid Kamal, ${ }^{1}$ Muhammad H. Zaman, ${ }^{2 \star}$ and Faisal Sultan ${ }^{1}$ \\ ${ }^{1}$ Shaukat Khanum Memorial Cancer Hospital and Research Centre, Lahore, Pakistan; ${ }^{2}$ Department of Biomedical Engineering, Boston University, \\ Boston, Massachusetts
}

\begin{abstract}
Pakistan is experiencing the first known outbreak of extensively drug-resistant (XDR) Salmonella enterica serotype Typhi (resistant to third-generation cephalosporins). The outbreak originated in Hyderabad in 2016 and spread throughout the Sindh Province. Whereas focus has remained on Sindh, the burden of XDR typhoid in Punjab, the most populous province, and the rest of the country is understudied. Using laboratory data from Shaukat Khanum Memorial Cancer Hospital and Research Centre in Lahore (Punjab Province) and its network of more than 100 collection centers across the country, we determined the frequency of blood culture-confirmed XDR typhoid cases from 2017 to 2019 . We observed an increase in XDR typhoid cases in Punjab, with the percent of ceftriaxone resistance among Salmonella Typhi cases increasing from no cases in 2017 , to $30 \%$ in 2018, and to $50 \%$ in 2019 , with children bearing the largest burden. We also observed spread of XDR typhoid to the two other provinces in Pakistan. To assess prevailing knowledge and practices on XDR typhoid, we surveyed 321 frontline healthcare workers. Survey results suggested that inappropriate diagnostic tests and antibiotic practices may lead to underdiagnosis of XDR typhoid cases, and potentially drive resistance development and spread. Of those surveyed, only $43.6 \%$ had heard of XDR typhoid. Currently, serological tests are more routinely used over blood culture tests even though blood culture is imperative for a definitive diagnosis of typhoid fever. We recommend stronger liaisons between healthcare providers and diagnostic laboratories, and increased promotion of typhoid vaccination among healthcare workers and the general population.
\end{abstract}

\section{INTRODUCTION}

Typhoid fever is a life-threatening bacterial infection caused by Salmonella enterica serotype Typhi. Recent reports by the WHO estimate up to 21 million cases and 161,000 deaths due to typhoid fever each year. ${ }^{1}$ Typhoid fever is typically contracted by drinking or eating Salmonella Typhi in contaminated food or water, and thus countries with poor or weak infrastructure and sanitation have a higher burden. ${ }^{2}$ Symptoms of typhoid fever include a prolonged high fever, headache, and gastrointestinal issues. Paratyphoid fever, which is similar but often less severe, is caused by Salmonella Paratyphi A, B, or C. ${ }^{1}$

Multidrug-resistant (MDR) typhoid strains have been endemic in Pakistan for the last two decades. ${ }^{3-5}$ In November 2016, an outbreak of extensively drug-resistant (XDR) typhoid cases was first identified in the Sindh Province of Pakistan. These were caused by MDR S. Typhi that had become resistant to third-generation cephalosporins and ciprofloxacin but were typically still susceptible to azithromycin and carbapenems. ${ }^{4}$ Early geospatial mapping revealed large clusters of ceftriaxone-resistant $S$. Typhi infections around sewage lines in the city of Hyderabad in the Sindh Province. ${ }^{6}$ Genomic sequencing revealed that isolates of a common MDR typhoid haplotype $\mathrm{H} 58$ acquired a plasmid with a bla $\mathrm{b}_{C T X-M-15}$ extended spectrum $\beta$-lactamase and qnrS fluoroquinolone resistance gene. ${ }^{4}$ The $\mathrm{H} 58$ isolates previously had chromosomally integrated a composite transposon carrying resistance genes to chloramphenicol, ampicillin, trimethoprim-sulfamethoxazole, and streptomycin, and a single mutation in gyrA which conferred intermediate susceptibility to ciprofloxacin.

\footnotetext{
*Address correspondence to Muhammad H. Zaman, Department of Biomedical Engineering, Boston University, 44 Cummington Mall, Boston, MA 02215. E-mail: zaman@bu.edu
}

†These authors contributed equally to this work.
The number of XDR typhoid cases in the province of Sindh has continued to grow. As of 2019, 15,284 cases from 23 districts have been reported since 2016, with 11,028 of these cases from the Karachi district. ${ }^{7-9}$ Current prevention strategies include vaccination programs, increasing surveillance and awareness, and improving sanitation and hygiene. Pakistan has four provinces (Figure 1), and although national and international emphasis on tracking the XDR typhoid has been focused on Sindh, spread in the other provinces has been observed. However, the extent of spread in the rest of Pakistan remains poorly understood. ${ }^{10,11}$ Thus, although the problem of XDR typhoid fever began in Hyderabad, in the Sindh Province (Southeast, Figure 1), this study tracked the spread of XDR typhoid cases in Pakistan, outside the Sindh Province. To do this, we examined blood culture data from Shaukat Khanum Memorial Cancer Hospital and Research Centre (SKMCH\&RC) in Lahore, Pakistan, and its extended network of more than 100 laboratory collection centers, which are predominately in the Punjab Province but spread across the country (North, Figure 1). To assess prevailing knowledge and practices, we also surveyed 321 frontline healthcare workers in four major cities, namely, Lahore, Faisalabad, Multan, and Peshawar, with the first three cities in Punjab and Peshawar being the capital and the largest city of the northwest Khyber Pakhtunkhwa (KPK) Province.

Here, we report the extensive rise of XDR typhoid cases in the province of Punjab and throughout Pakistan. We also explore gaps in knowledge and compromised practices which may contribute to underreporting and poor management of XDR typhoid, leading to the development of antibiotic resistance and spread.

\section{METHODS}

Sample collection. Retrospective patient data analysis was conducted at SKMCH\&RC and comprised microbiological susceptibility data between January 2017 and December 


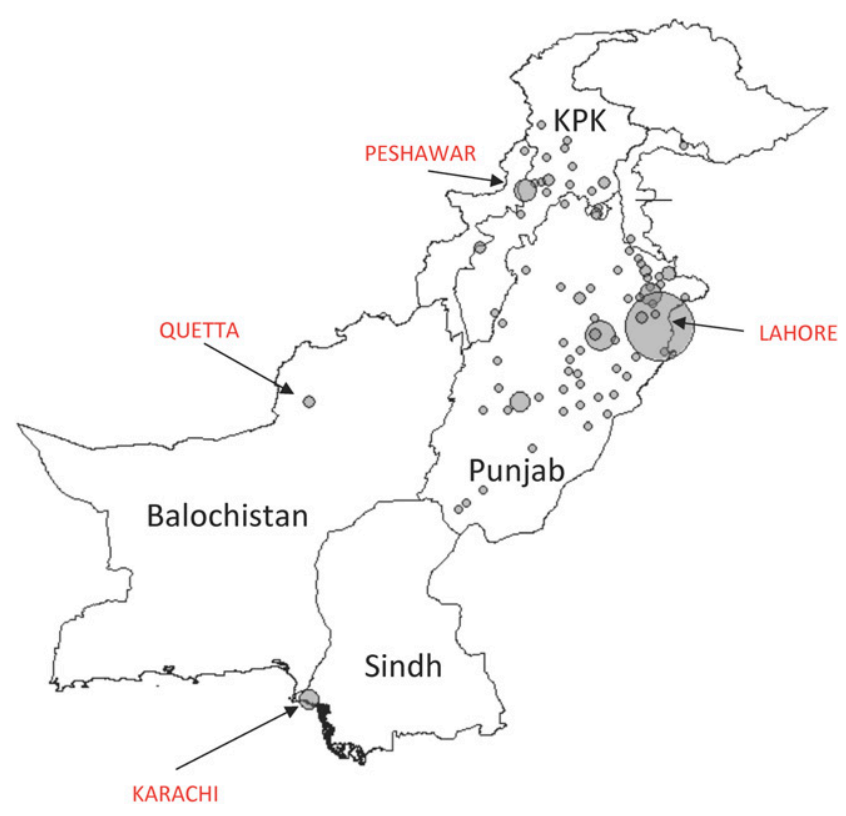

FIGURE 1. Distribution of Shaukat Khanum Memorial Cancer Hospital and Research Centre collection centers across Pakistan. The more collection centers within a city, the bigger the area of the circle. The four provinces (Sindh, Punjab, Balochistan, and Khyber Pakhtunkhwa [KPK]) and major cities are labeled. This figure appears in color at www.ajtmh.org.

2019 from SKMCH\&RC and the hospital's extended network of laboratory collection centers across Pakistan (Figure 1). Locations of collection centers were plotted onto a map of Pakistan using $R$ studio and the "maps" package. ${ }^{12,13}$ These collection centers are independently and strategically located in areas close to general hospitals or private clinics. All data was retrieved from the online records on the in-house information system database. We define ceftriaxone-resistant S. Typhi as XDR.

Antimicrobial susceptibility testing/bacterial identification. All blood culture samples collected via the SKMCH\&RC's laboratory network were cultured using the BacT/Alert blood culture system (bioMérieux, Marcy-l'Étoile, France). Positive signaled bottles were subcultured onto chocolate, blood, and MacConkey agar plates. Colonies suggestive of Salmonellae were identified based on colony morphology, serotyping, and biochemical tests using API 20E (bioMérieux). Salmonella isolates were tested for antibiotic susceptibility by Kirby-Bauer disc diffusion method on Muller-Hinton agar with standard antimicrobial discs. ${ }^{14}$ Salmonella isolates were tested for ampicillin, ceftriaxone, chloramphenicol, co-trimoxazole, and ciprofloxacin susceptibility in accordance with Clinical Laboratory Standards Institute (CLSI) guidelines of the respective year. ${ }^{15}$ We calculated positivity rates of $S$. Typhi/Paratyphi A using the number of positive cases from the total number of blood cultures performed each year. ${ }^{5}$

Survey questionnaire. A total of four continuing medical education (CME) sessions were conducted for doctors and healthcare workers, in the form of seminars, in Lahore, Faisalabad, Multan, and Peshawar between August and December 2018. Before discontinuing the provision of serological tests, namely, Typhidot and Widal, CME sessions were held to increase awareness regarding the emerging XDR problem, to promote blood culture, and to inform healthcare professionals that serological tests would no longer be offered by the
SKMCH\&RC laboratory services and blood cultures should be sent instead. During these CME sessions, we also administered a questionnaire of knowledge and best practices for treating S. Typhi infections. A total of 321 healthcare workers (trainees, medical officers, and general practitioners) were surveyed. The survey questionnaire (Supplemental Table S1) included directed prompt questions.

Patient and public involvement. Patients or the public were not involved in the design, data analysis, or manuscript preparation of our research. The results of the study will be disseminated when possible.

\section{RESULTS}

Salmonella Typhi/Paratyphi A positivity rates have increased from 2017 to 2019 . We previously reported a decrease in typhoid positivity rates from 1992 to $2015 .^{5}$ To investigate if there were any changes to this trend, we determined positivity rates for $S$. Typhi and $S$. Paratyphi A from 2017 to 2019 . The contribution of $S$. Typhi to total blood cultures performed increased from $0.6 \%$ in $2017(n=16,429)$, to $1 \%$ in $2018(n=17,694)$, and to $4.3 \%$ in $2019(n=36,003)$ (Figure 2). Positivity rates for $S$. Paratyphi $A$ increased from $0.2 \%$ in 2017 , to $0.3 \%$ in 2018, and to $0.9 \%$ in 2019 (Figure 2). Overall, this suggests that a previously reported decline in typhoid incidence ${ }^{5}$ is shifting upward.

Prevalence of XDR typhoid fever has increased in Punjab and countrywide, indicating spread outside Sindh. In 2017 , we received 16,429 blood cultures, of which 2,735 were positive for bacterial or fungal growth. $S$. Typhi was identified in 93 of these blood cultures, of which only two cases $(2.2 \%)$ were found to be XDR (Figure $3 A$ ). Both of these blood cultures were from children aged $<5$ years submitted in Quetta, Balochistan. In 2018, we received 17,694 blood cultures, 2,817 of which were positive for bacterial or fungal growth. Salmonella Typhi was identified in 175 of these cases, of which $50(28.6 \%)$ were found to be XDR (Figure 3A). In 2019, we received 36,003 blood cultures, of which 6,116 were positive for bacterial or fungal growth. Of the 1,535 reported $S$. Typhi cases, 783 (51.0\%) were XDR (Figure 3A). Further antibiotic susceptibility data for Salmonella Typhi, based on CLSI reporting guidelines, indicated high levels of resistance to ciprofloxacin, ampicillin, and chloramphenicol (Table 1,

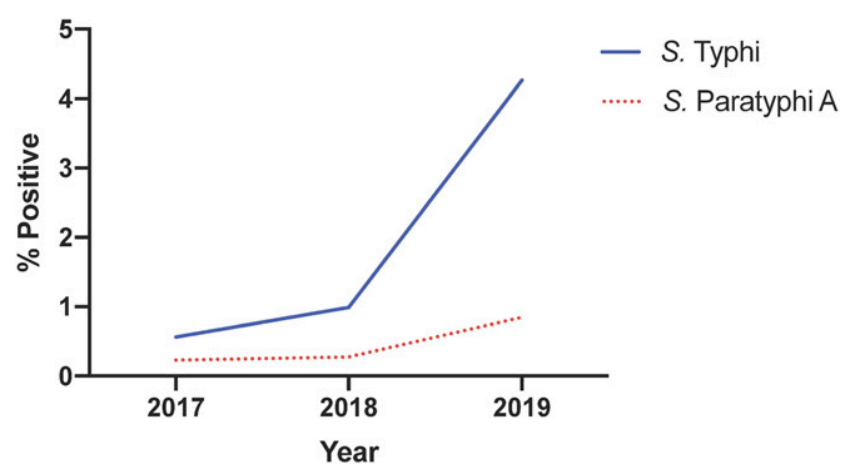

FIGURE 2. Positivity rates for Salmonella Typhi and Salmonella Paratyphi A from Shaukat Khanum Memorial Cancer Hospital and Research Centre, calculated as \% of total number of blood cultures performed each year that were positive for S. Typhi or S. Paratyphi A. This figure appears in color at www.ajtmh.org. 

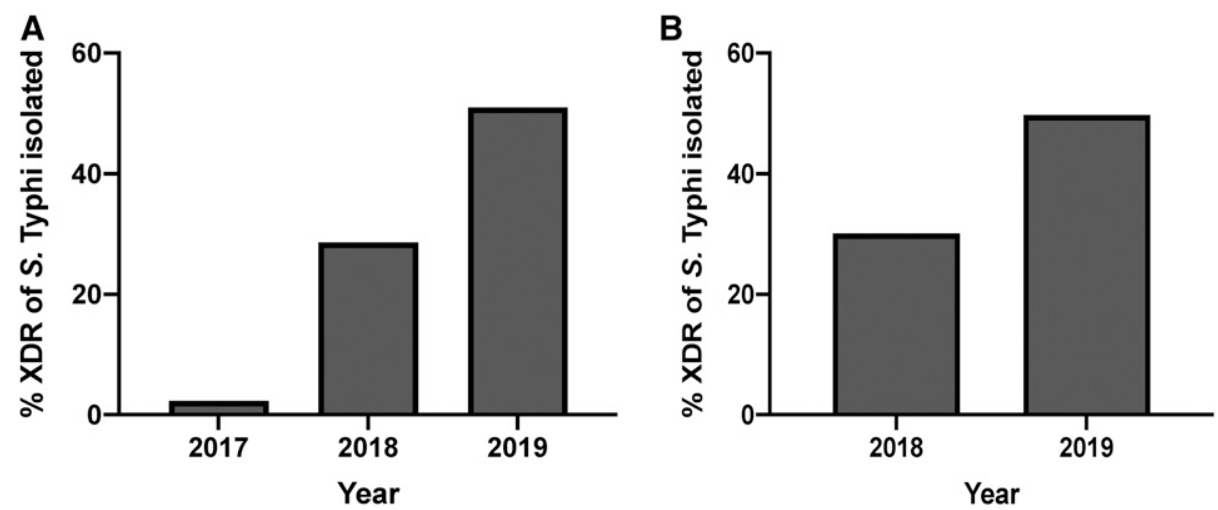

Figure 3. (A) \% of all Salmonella Typhi isolates from blood culture samples that were XDR and (B) $\%$ of all S. Typhi isolates from blood culture samples that were from Punjab from 2017 to 2019 for Shaukat Khanum Memorial Cancer Hospital and Research Centre. For (B), there were no detected cases of XDR typhoid in 2017. XDR = extensively drug-resistant.

Figure 4A). Susceptibility data against ciprofloxacin demonstrate a marked switch from intermediate to resistant from 2017 to 2019, which is another marker of XDR typhoid (Figure 4A). Azithromycin, meropenem, and imipenem susceptibility was also assayed, and no resistance was observed in any of the XDR Salmonella Typhi isolates in the years 2017, 2018, and 2019.

Breaking down the large number of XDR S. Typhi cases from 2019 by region, we observed incidences across the country (Table 2). A majority of the XDR typhoid cases were in Punjab (Figure 1) and hence demonstrates a widespread incidence of XDR typhoid in the region. In Punjab, of all the $S$. Typhi cases, the proportion that were XDR increased from no cases isolated in $2017(n=76)$, to $30.1 \%(n=156)$ in 2018, and to 49.8\% ( $n=1,369)$ in 2019 (Figure 3B). A full breakdown of cases by district for Punjab is provided in Supplemental Table S2. We observed a higher burden of XDR typhoid cases in children aged 6 years and younger (40.3\%, Figure 4B) in Punjab, which is similar to initial findings in Sindh. ${ }^{6}$

Healthcare provider experience with typhoid fever, XDR typhoid, and best practices for vaccination. We surveyed 321 healthcare workers (trainees, medical officers, and general practitioners) from Lahore, Faisalabad, Peshawar, and Multan about knowledge and practices related to the management of typhoid fever. Although a majority of healthcare workers saw patients with typhoid fever (62.6\%), only $43.6 \%$ of respondents had heard of XDR typhoid. Table 3 presents the most characteristic symptoms of typhoid fever from healthcare provider experience. Providers reported that the most common symptoms among their patients with typhoid fever were high-grade fever (75.4\%), followed by abdominal pain $(73.2 \%)$. Of all respondents, $17.8 \%$ routinely advised vaccination and only $12.8 \%$ were vaccinated themselves against typhoid. Full survey results are provided in Supplemental Table S1.

Diagnosis and treatment of typhoid fever. Whereas $47 \%$ of respondents routinely sent serological tests for the diagnosis of typhoid, only $31.5 \%$ routinely sent blood cultures. The choice for timing of sending blood cultures was varied, with the first visit if there is a suspicion of typhoid fever at $34.6 \%$, before the start of antibiotics at $37.7 \%$, and when the fever does not defervesce after 48 hours of antibiotics at $33.0 \%$. Only $5.3 \%$ responded after the first dose of antibiotics. Once blood culture is performed, $78.8 \%$ modify therapy on the basis of blood culture susceptibility data. Table 4 presents respondents' empirical choice of antibiotic when a patient first presents with the suspicion of typhoid fever. Ceftriaxone (34\%) was the most popular empirical choice of antibiotic for those with suspected typhoid fever, followed by ciprofloxacin $(26.8 \%)$.

\section{DISCUSSION}

We report that the prevalence of culture-confirmed typhoid is increasing (Figure 2) and that XDR typhoid is now widespread in the Punjab Province of Pakistan, indicating spread beyond the southern province of Sindh, where the outbreak began (Figure 3). Thus, without appropriate diagnostics, interventions, or surveillance, XDR typhoid will continue to increase and remain underreported. Given the stress on the health system due to COVID-19, the additional and preexisting burden of XDR typhoid is likely to worsen without active and robust interventions. In addition, many symptoms of COVID19 are similar to typhoid, which increases the risk of misdiagnosis. ${ }^{11}$ For 2019, from our dataset, there were 682 XDR typhoid cases among 30 districts of Punjab, with the majority in the Lahore district (495 cases). This number is concerning as

TABLE 1

Antibiotic susceptibility profile of Salmonella Typhi isolated in 2017, 2018, and 2019

\begin{tabular}{|c|c|c|c|c|c|}
\hline \multirow[b]{2}{*}{ Year } & \multicolumn{5}{|c|}{$\%$ Resistant } \\
\hline & Ampicillin & Chloramphenicol & Co-trimoxazole & Ciprofloxacin (intermediate) & Ciprofloxacin (resistant) \\
\hline $2017(n=93)$ & 51 & 55 & 52 & 74 & 19 \\
\hline $2018(n=175)$ & 70 & 62 & 59 & 69 & 26 \\
\hline $2019(n=1,535)$ & 67 & 63 & 64 & 40 & 58 \\
\hline
\end{tabular}



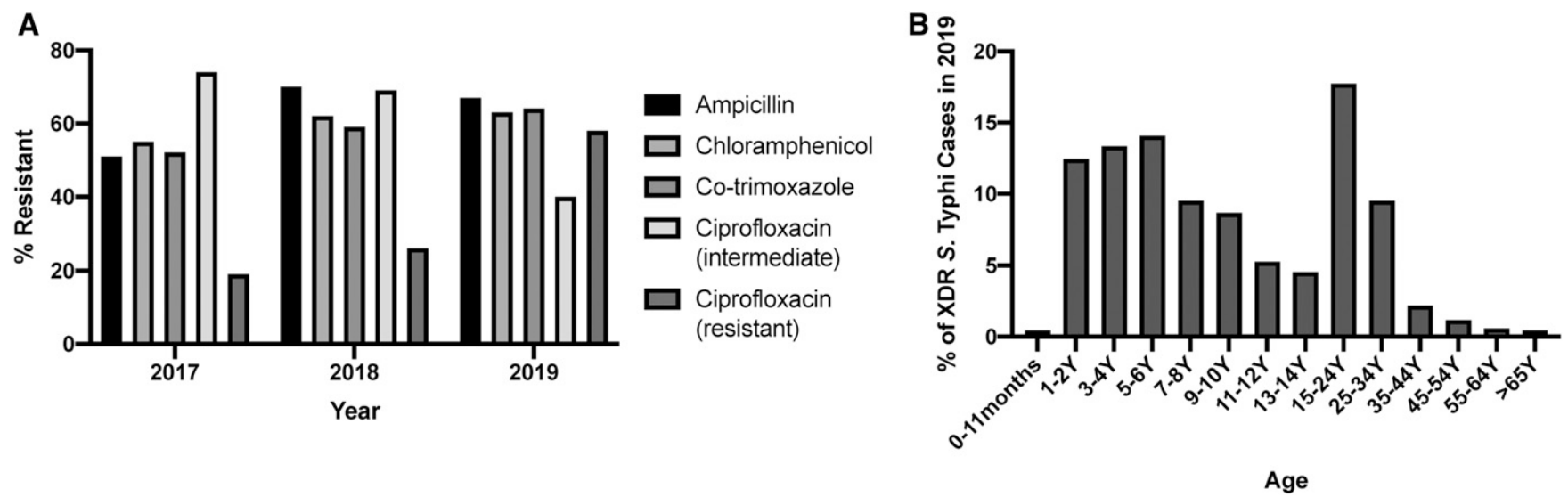

Figure 4. (A) \% resistant to ampicillin, chloramphenicol, co-trimoxazole, and ciprofloxacin (intermediate or resistant) of $S$. Typhi isolated from blood culture samples in 2017, 2018, and 2019, and (B) age distribution of all extensively drug-resistant (XDR) S. Typhi cases isolated in 2019 from Shaukat Khanum Memorial Cancer Hospital and Research Centre from Punjab $(n=682)$.

Sindh reported a similar 664 XDR cases in 2017, which increased to 4,810 in $2018,{ }^{9}$ although this may also reflect increased testing and surveillance. Notably, we also report XDR typhoid in Balochistan and KPK, suggesting that the outbreak has reached and permeated all corners of the country (Table 2, Figure 1). We also find that the burden is highest in children (Figure 4B).

We surveyed knowledge and practices among healthcare workers in Punjab and KPK, and found that XDR typhoid cases are likely to be underreported because of lack of blood culture tests with antimicrobial susceptibility data. In 2017, the Pakistani National Action Plan recommended that serological tests for the detection of typhoid antibody be replaced by blood culture tests. ${ }^{16}$ Blood culture tests provide definitive diagnosis and antimicrobial susceptibility data, ${ }^{17}$ thus providing accurate case counts and reducing inappropriate antibiotic use. ${ }^{18,19}$ However, we found that only $31.5 \%$ of healthcare providers routinely use blood culture, indicating that increased awareness is needed (Supplemental Table S1). Although we had observed an increase in XDR typhoid cases, switching to blood culture from serological tests to identify typhoid in our laboratories led us to strengthen our observation and better identify the scope of the problem which highlights the value of improved diagnostic choices. Although there are no national guidelines for typhoid treatment, the Medical Microbiology and Infectious Diseases Society of Pakistan has published guidelines and recommendations to improve practice and treatment to fill the gap in available recommendations from international agencies and help doctors improve practices and better handle the XDR typhoid outbreak. ${ }^{20}$

TABLE 2

Regional break up of ceftriaxone-resistant S. Typhi isolated in 2019

\begin{tabular}{lc}
\hline \multicolumn{1}{c}{ Province/region } & $\begin{array}{c}\text { Number of extensively } \\
\text { drug-resistant cases isolated }\end{array}$ \\
\hline Azad Jammu and Kashmir & 3 \\
Federally administered tribal areas & 4 \\
Sindh & 8 \\
Khyber Pakhtunkhwa & 18 \\
Balochistan & 68 \\
Punjab & 682 \\
Total & 783 \\
\hline
\end{tabular}

Increased efforts are necessary to improve awareness about XDR typhoid among the general population and healthcare workers such that patients can ask for appropriate diagnostics and healthcare workers understand the importance of appropriate diagnostics and treatment. Currently, lack of knowledge may force patients to choose more economical tests or alternatives to diagnostics. We stress that in cases of suspected typhoid fever, serological tests should be avoided and blood culture should be sent before starting antibiotic therapy instead, to avoid treatment failure or inappropriate antibiotic usage. Furthermore, laboratories must strengthen their infrastructure and quality control and assurance programs and ensure that standardized protocols and guidelines are implemented and followed. These measures will also allow for improved surveillance of $S$. Typhi cases and reducing the risk of developing bacterial antibiotic resistance due to inappropriate antibiotic usage. There should also be improved liaison between healthcare providers and diagnostic laboratories. In Pakistan, antibiotics are available without prescription and self-medication with antibiotics is common. ${ }^{21-23}$ In addition, cases of ceftriaxone-resistant $S$. Typhi (Figure 2) are increasing, whereas the top empirical choice among healthcare providers for treatment is currently ceftriaxone (Table 4) -creating a dangerous scenario in which ineffective antibiotics are frequently used. Thus, given the growing outbreak, availability of antibiotics over the counter

TABLE 3

In your experience, which of the following are the most characteristic symptoms of typhoid fever? (Can choose multiple)

\begin{tabular}{lcc}
\hline \multicolumn{1}{c}{ Symptom } & No. of responses & $\%(n=321)$ \\
\hline High-grade fever & 242 & 75.4 \\
Abdominal pain & 235 & 73.2 \\
Aches and pains & 183 & 57.0 \\
Nausea & 170 & 53.0 \\
Fatigue & 158 & 49.2 \\
Headache & 154 & 48.0 \\
Vomiting & 152 & 47.4 \\
Diarrhea & 140 & 43.6 \\
Constipation & 128 & 39.9 \\
Cough & 54 & 16.8 \\
Jaundice & 44 & 13.7 \\
\hline
\end{tabular}


TABLE 4

What is your empirical choice of antibiotic when a patient first presents with the suspicion of typhoid fever?

\begin{tabular}{lcc}
\hline \multicolumn{1}{c}{ Antibiotic } & No. of responses & $\%(n=321)$ \\
\hline $\begin{array}{l}\text { Ceftriaxone/cefixime/third-generation } \\
\quad \text { cephalosporin }\end{array}$ & 109 & 34.0 \\
Ciprofloxacin & 86 & 26.8 \\
$\begin{array}{l}\text { Azithromycin } \\
\text { Third-generation cephalosporin + } \\
\quad \text { ciprofloxacin }\end{array}$ & 24 & 7.5 \\
Azithromycin + ciprofloxacin & 14 & 4.4 \\
$\begin{array}{l}\text { Third-generation cephalosporin + } \\
\quad \text { azithromycin }\end{array}$ & 5 & 1.6 \\
No response & 5 & 1.6 \\
\hline
\end{tabular}

and ineffective empiric antibiotic treatment, it will be important to revisit which antibiotics remain effective against typhoid and which do not, or to develop new combination therapies to manage cases. This can include expanding the panel of antibiotics to test Salmonella against to determine if it is preferable to return to older antibiotics.

We found that vaccine advocacy was very low among healthcare workers (Supplemental Table S1). Undervaccination in Pakistan is a problem, in general due to issues with logistics, awareness, education, and healthcare worker attitudes. ${ }^{24}$ The WHO prequalified the new conjugate typhoid vaccine (Typbar TCV, Bharat Biotech, Hyderabad, India) in January 2018 and recommended use in March 2018 in children aged 6 months or older in countries endemic with typhoid. ${ }^{18}$ Use of the typhoid vaccine can lower typhoid incidence and subsequently reduce unnecessary antibiotic use which can drive resistance. ${ }^{19}$ In November 2019, Pakistan launched the typhoid vaccine into its routine Expanded Program of Immunization (EPI) in Sindh for children aged 9 months -15 years. ${ }^{25}$ Because of supply, the vaccine was planned to be introduced in Punjab Province in 2020 and nationally in 2021; however, introduction in Punjab has been pushed back because of COVID-19 and supply issues (personal communication). ${ }^{25}$ Thus, the limited supply in Pakistan is a hurdle to advising vaccinations. Furthermore, trust in vaccinations is another barrier, ${ }^{24}$ and although introduced into the EPI for children, there is no program for adults, who may be unlikely to self-advocate for vaccination with out-of-pocket costs or unless persuaded by a provider. Thus, the role of the healthcare provider in education and advocacy is critical to build trust for the typhoid vaccine in the general population. However, first, more work needs to be done to ensure availability and access of the vaccine and build trust and awareness of the typhoid vaccine among healthcare workers themselves. Overall, steps highlighted in the Discussion earlier can help mitigate the outbreak of XDR typhoid in Pakistan.

Received July 1, 2020. Accepted for publication December 13, 2020.

Published online January 25, 2021.

Note: Supplemental tables appear at www.ajtmh.org.

Acknowledgments: We would like to thank the Microbiology and IT staff of SKMCH\&RC for their assistance.

Disclosure: This study was granted exemption from the SKMCH\&RC Institutional Review Board.

Authors' addresses: Summiya Nizamuddin, Department of Pathology, Shaukat Khanum Memorial Cancer Hospital and Research Centre, Lahore, Pakistan, E-mail: summiyan@skm.org.pk. Rashid Kamal and
Faisal Sultan, Department of Medicine, Shaukat Khanum Memorial Cancer Hospital and Research Centre, Lahore, Pakistan, E-mails: rashidkamal@skm.org.pk and faisal@skm.org.pk. Carly Ching and Muhammad H. Zaman, Department of Biomedical Engineering, Boston University, Boston, MA, E-mails: chingc@bu.edu and zaman@ bu.edu.

\section{REFERENCES}

1. World Health Organization, 2018. Typhoid. Geneva, Switzerland: WHO. Available at: https://www.who.int/immunization/diseases/ typhoid/en/. Accessed April 4, 2020.

2. Dougan G, Baker S, 2014. Salmonella enterica serovar typhi and the pathogenesis of typhoid fever. Annu Rev Microbiol 68: 317-336.

3. World Health Organization, 2020. Emergencies Preparedness, Response Typhoid Fever - Islamic Republic of Pakistan. Geneva, Switzerland: WHO. Available at: https://www.who.int/csr/ don/27-december-2018-typhoid-pakistan/en/. Accessed April 4, 2020.

4. Klemm EJ et al., 2018. Emergence of an extensively drug-resistant Salmonella enterica serovar typhi clone harboring a promiscuous plasmid encoding resistance to fluoroquinolones and thirdgeneration cephalosporins. mBio 9: e00105-e00118.

5. Das JK, Hasan R, Zafar A, Ahmed I, Ikram A, Nizamuddin S, Fatima S, Akbar N, Sultan F, Bhutta ZA, 2018. Trends, associations, and antimicrobial resistance of Salmonella typhi and paratyphi in Pakistan. Am J Trop Med Hyg 99: 48-54.

6. Qamar FN et al., 2018. Outbreak investigation of ceftriaxoneresistant Salmonella enterica serotype Typhi and its risk factors among the general population in Hyderabad, Pakistan: a matched case-control study. Lancet Infect Dis 18: 1368-1376.

7. Field Epidemiology and Disease Surveillance Division, 2020. Seasonal Awareness and Alert Letter. Islamabad, Pakistan: National Institute of Health.

8. World Health Organization, 2019. Weekly epidemiological monitor. World Health Organ 6: 22765273.

9. Field Epidemiology and Disease Surveillance Division, 2020. Weekly Field Epidemiology Report. Islamabad, Pakistan: National Institute of Health.

10. Saeed N, Usman M, Khan EA, 2019. An overview of extensively drug-resistant Salmonella typhi from a tertiary care hospital in Pakistan. Cureus 11: e5663.

11. Haqqi A, Khurram M, Din MSU, Aftab MN, Ali M, Ahmed H, Afzal MS, 2020. COVID-19 and Salmonella Typhi co-epidemics in Pakistan: a real problem. J Med Virol. doi: 10.1002/jmv.26293.

12. RCore Team, 2013. R: A Language and Environment for Statistical Computing. Vienna, Austria: R Foundation for Statistical Computing. Available at: http://www.r-project.org/. Accessed June 2, 2020.

13. Becker RA, Wilks AR, Minka TP, Deckmyn A, 2018. Maps: Draw Geographical Maps. R Package Version 3.3.0. Available at: https://cran.r-project.org/package=maps. Accessed June 2, 2020.

14. Bauer AW, Kirby WMM, Sherris JC, Turck MD, 1966. Antibiotic susceptibility testing by a standardized single disk method. Am J Clin Pathol 45: 493-496.

15. CLSI, 2018. Performance Standards for Antimicrobial Susceptibility Testing. 28th ed. CLSI supplement M100. Wayne, PA: Clinical and Laboratory Standards Institute.

16. Ministry of National Health Services Regulations \& Coordination Government of Pakistan, 2017. Antimicrobial resistance national action plan Pakistan. Antimicrob Resist 6: 1-174.

17. World Health Organization, 2003. Background document: the diagnosis, treatment and prevention of typhoid fever. Commun Dis Surveill Response Vaccin Biol: 1-38.

18. Andrews JR, Qamar FN, Charles RC, Ryan E, 2018. Extensively drug-resistant typhoid - are conjugate vaccines arriving just in time? N Engl J Med 379: 1491-1493.

19. Andrews JR et al., 2019. Typhoid conjugate vaccines: a new tool in the fight against antimicrobial resistance. Lancet Infect Dis 19: e26-e30.

20. Medical Microbiology and Infectious Diseases Society of Pakistan, 2019. Typhoid Management Guidelines 2019. Karachi, 
Pakistan: The Medical Microbiology and Infectious Diseases Society of Pakistan, 1-12. Available at: https://www.mmidsp.com/ typhoid-management-guidelines-2019/. Accessed April 7, 2020.

21. Nazir S, Azim M, 2017. Assessment of antibiotic self-medication practice among public in the northwestern region of Pakistan. Eur J Hosp Pharm 24: 200-203.

22. Gillani AH, Ji W, Hussain W, Imran A, Chang J, Yang C, Fang Y, 2017. Antibiotic self-medication among non-medical university students in Punjab, Pakistan: a cross-sectional survey. Int $J$ Environ Res Public Health 14: 1152.

23. Saleem $Z$ et al., 2020. Sale of WHO AWaRe groups antibiotics without a prescription in Pakistan: a simulated client study. J Pharm Policy Pract 13: 26.
24. Butt M, Mohammed R, Butt E, Butt S, Xiang J, 2020. Why have immunization efforts in Pakistan failed to achieve global standards of vaccination uptake and infectious disease control? Risk Manag Healthc Policy 13: 111-124.

25. World Health Organization, 2019. Pakistan Becomes First Country to Introduce New Typhoid Vaccine into Routine Immunisation Program. Geneva, Switzerland: WHO. Available at: http:// www.emro.who.int/pak/pakistan-news/pakistan-first-country-tointroduce-new-typhoid-vaccine-into-routine-immunizationprogramme.html\#: :text=Pakistan-, Pakistan first country to introduce new typhoid vaccine into routine, into its routine immunization. Accessed November 24, 2020. 